\title{
7 Intervenção com mães de crianças hospitalizadas
}

\author{
| Carla Ferreira ${ }^{1}$ |
}

\section{RESUMO}

O presente trabalho teve como objectivo implementar intervenções de ajuda/apoio emocional a 47 mães que acompanhavam os seus filhos hospitalizados, durante um período de dezassete meses (Janeiro 2009/Maio 2010).

As mães foram referenciadas pela equipa de Enfermagem do Serviço de Pediatria, após detecção de alterações do seu estado emocional, nomeadamente a nível da ansiedade, depressão e stresse. $\mathrm{O}$ apoio emocional prestado às mães, reforçou a adaptação destas no processo de doença e hospitalização do seu filho. As intervenções desenvolvidas foram no sentido de fornecer apoio, atenção, compreensão, suporte, clarificação dos sentimentos e escuta com as mães.

O desenvolvimento desta intervenção permitiu construir uma reflexão sobre aspectos que tornam a presença dos pais inequivocamente essencial no que é hoje a assistência à criança hospitalizada e da necessidade de cuidados às famílias, nomeadamente a nível do apoio emocional.

\section{PALAVRAS-CHAVE: Mães de Crianças Hospitalizadas; Relação de Ajuda; Enfermagem de Ligação.}

\section{ABSTRACT}

This study aimed to help implement interventions / emotional support to 47 mothers who accompanied their children hospitalized for a period of seventeen months (January 2009/ May 2010), at de Department of Pediatrics of the Santarem's Hospital.

Mothers were referred to by the nursing staff after detection of changes in their emotional state, particularly at the level of anxiety, depression and stress. The emotional support provided to mothers, reinforced the adaptation of these strengthened in the process of illness and hospitalization of her son. The interventions were developed in order to provide support, care, understanding, support, clarification of feelings and listen with their mothers.
The development of this intervention led to a reflection on aspects that make the presence of parents clearly essential in what is now the care of hospitalized children and the need to care for families, especially at the level of emotional support.

\section{KEY WORDS: Mothers of hospitalized children; Helping Relationship; Nursing Liaison.}

\section{INTRODUÇÃO}

A hospitalização de uma criança transporta consigo grandes mudanças na vida quer da criança, quer dos pais. A minimização desta mudança ou transição situacional no ciclo vital passa pela presença contínua dos pais durante as 24 horas do dia.

São indiscutíveis as vantagens da permanência dos pais durante a hospitalização da criança, quer para esta quer para aqueles. As mudanças verificadas implicam adaptações dos pais a uma nova realidade que só será ajustada de forma equilibrada se o hospital oferecer condições físicas e humanas para isso. Estas condições passam por um acolhimento capaz de dar respostas às suas necessidades perspectivando uma continuidade do papel parental.

O modo como a família se adapta à situação de ter uma criança doente e hospitalizada, depende dos seus próprios recursos intrínsecos, bem como de suportes familiares e sociais disponíveis. A atenção passa por conhecer os seus medos, dificuldades, sentimentos e necessidades, respeitando o conhecimento parental da criança e o seu direito a participar na tomada de decisões e no processo de prestação de cuidados.

Encontramos como respostas comuns dos pais da criança hospitalizada, a negação (habitualmente reacções iniciais), sentimentos de culpa, medo e ansiedade (relacionados com a gravidade da doença e tipo de procedimentos/ tratamentos necessários e consequente trauma e dor que passam provocar na criança), depressão (que pode surgir após a crise aguda e recuperação total, sendo muitas vezes acompanhada de exaustão física e mental), impotência, revolta, e intensificação de problemas preexistentes (Bicho

\footnotetext{
${ }^{1}$ Enfermeira Especialista em Enfermagem de Saúde Mental e Psiquiatria, Serviço de Hospital de Dia do Departamento de Psiquiatria e Saúde Mental do Hospital de Santarém, carla.esmp@gmail.com

Submetido em 08-03-2011. Aceite em 27-05-2011.

Citação: Ferreira, C. (2011). Intervenção com mães de crianças hospitalizadas. Revista Portuguesa de Enfermagem de Saúde Mental. 5, 45-51.
} 
\& Pires, 2002 ao citar Cárter), tudo isto associado a níveis elevados de stress. Estas reacções ocorrem porque, segundo Schmitz (in Bicho \& Pires, 2002), são muitos os problemas que os pais enfrentam perante a hospitalização de um filho: medo da doença e do desconhecido; ambivalência para com o filho; ausência de controlo em relação ao meio hospitalar (pessoas, rotinas e equipamentos); mudanças de hábitos de vida e no atendimento das necessidades do filho doente e dos outros filhos; insegurança quanto aos tratamentos e seus resultados; problemas financeiros, de emprego e outros de natureza social e por último padrões comportamentais solicitados, diferentes dos habituais.

Reis \& Santos (1996), consideram que os cuidados centrados na família, prestados em parceria com esta, são a filosofia da enfermagem, sendo o Enfermeiro Especialista de Saúde Mental e Psiquiatria o mais apto para tal. As crenças e valores que sustentam essa filosofia incluem o reconhecimento de que os pais são os melhores prestadores de cuidados à criança. Para que os pais sejam um elemento efectivo da equipa assistencial, necessitam ser ajudados desde o primeiro momento na realização do seu papel dentro da mesma (Jorge, 2004).

Os defensores da humanização do atendimento (Moleiro, 1991; Reis \& Santos, 1996; Marinheiro, 2002) na saúde/ doença, preconizam a presença do Enfermeiro de Ligação "todos os escritos de autores interessados (...) acentuam o papel da enfermeira (...) como alguém que ajude e trate a família e a pessoa toda de cada um" (Gomes, 1999: 153). A presença do enfermeiro de ligação da área da saúde mental e psiquiatria é incontestavelmente uma mais-valia para a equipa multidisciplinar, quer em termos directos destes profissionais na sua prática dos cuidados, quer na colaboração que é prestada à equipa na prestação dos cuidados (Mota, 2000).

Neste sentido, efectuaram-se intervenções de ajuda às mães durante a hospitalização da criança, tendo como finalidade o apoio emocional. Estas tiveram um carácter individual, assentes na criação de uma relação de confiança e empática, onde se pretendeu identificar e dissecar o problema precipitador da crise, identificar e implementar recursos emocionais, familiares, entre outros, que melhorassem o funcionamento emocional face à situação.

Este tipo de intervenção, pretende contribuir para uma melhoria da qualidade dos cuidados em enfermagem, tendo como parceiros efectivos na prestação dos cuidados os pais/conviventes significativos e reforçar a importância do Enfermeiro de Ligação em Enfermagem de Saúde Mental no Serviço de Pediatria.

São objectivos deste trabalho:

- Avaliar as respostas humanas das mães face à hospitalização das crianças (ansiedade, depressão e stresse);
- Identificar a relação existente entre as características sócio-demográficas das mães e as respostas humanas face à hospitalização das crianças;

- Identificar a relação que existe entre as características das crianças e as respostas humanas das mães;

- Identificar a relação existente entre as características da hospitalização das crianças e as respostas humanas das mães.

\section{METODOLOGIA}

\section{Participantes}

Participaram neste estudo 47 mães que acompanharam os filhos hospitalizados. A média de idade das mães é de 33,17 anos $(\mathrm{DP}=6,66)$, variando entre os 16 anos e os 50 anos.

\section{Instrumentos}

Para dar cumprimento aos objectivos do trabalho, foi seleccionado o seguinte instrumento de colheita de dados:

- Caracterização da amostra em estudo que incluiu dados relativos aos pais e crianças: idade dos pais e crianças, condiçõessócio-demográficas (estado civil, sexo, habilitações literárias, idade, situação laboral), e familiares (numero de irmãos). Incluiu ainda o conhecimento das características da hospitalização (hospitalizações anteriores, inicio da doença e parentesco de quem acompanha a criança).

- Como instrumento de medida para o estudo foi utilizado a Escala de Ansiedade, Depressão e Stress (EADS) (Lovibond \& Lovibond, 1995) - Versão Portuguesa de Pais-Ribeiro, Honrado e Leal (2004). Trata-se de uma escala constituída por 21 itens, estando organizada em três sub-escalas: Depressão, Ansiedade e Stress, cada uma das quais constituída por sete itens. A pontuação total da escala é fornecida através dos resultados de 3 sub-escalas, variando de 0 (mínimo) a 21 (máximo), no qual resultados mais elevados significam maiores níveis de ansiedade, depressão e stresse.

\section{Procedimento}

A recolha de dados foi realizada entre Janeiro de 2009 e Maio de 2010, no Hospital de Santarém, nomeadamente, no Serviço de internamento de Pediatria. O questionário foi preenchido pelas mães que acompanhavam o filho(a) internado. Foi explicado a finalidade e os objectivos principais do estudo a cada participante, e, obtido o seu consentimento informado.

Os critérios de inclusão foram os seguintes:

- Pais que estivessem a acompanhar o filho durante a hospitalização; 
- Pais de crianças com idades compreendidas entre 1 mês de idade e os catorze (14) anos;

- Pais referenciados pela equipa de Enfermagem do Serviço.

\section{ANÁLISE DOS RESULTADOS}

\section{Características das Mães}

Relativamente à variável parentesco de quem acompanha a criança, incluída no instrumento de colheita de dados, não é efectuada qualquer referência a valores absolutos atendendo a que, durante o período de colheita de dados, somente as mães/mulheres acompanharam as crianças hospitalizadas. Apesar de a legislação prever a permanência de acompanhante da criança, independentemente do sexo, no período de recolha de dados estiveram presentes somente as mães o que não significa que estas crianças, ao longo da hospitalização, não usufruíssem também da presença do pai ou outros elementos significativos. A maior representatividade de indivíduos do sexo feminino no acompanhamento dos filhos hospitalizados, também foi confirmada no estudo de Jorge (2004). A autora afirma que culturalmente, continua, ainda, a ser atribuído um maior peso de responsabilidade à mulher, relativamente ao acompanhamento da criança nos momentos em que esta necessita de mais cuidados particularmente nos períodos de doença. Um outro estudo realizado por Pedro (2009), faz referência como sendo a mulher em $70 \%$ dos casos que assume a função de cuidados ao membro doente, no papel da mãe, fruto de influências culturais.

Constatou-se que 11 mães têm o $3^{\circ}$ Ciclo do ensino básico $(23,4 \%)$ e 10 mães concluíram o ensino superior (21,3\%). Com o $1^{\circ}$ Ciclo e $2^{\circ}$ ciclo, temos em igual número, 9 mães $(19,1 \%)$, e 8 mães com o secundário (17\%).

No que diz respeito ao estado civil, 40 são casadas ou vivem em união de facto $(85,1 \%)$, seis são divorciadas $(12,8 \%)$ e 1 é viúva $(2,1 \%)$.

Relativamente à residência, 27 mães (57,4\%) vivem em meio rural, as restantes, que corresponde a 20 mães $(42,6 \%)$ vivem em meio urbano.

Quanto à situação profissional, sobressai que, no momento da recolha dos dados, 27 mães (57,4\%) encontravam-se empregadas, estando as restantes desempregadas, 20 mães $(42,6 \%)$.

\section{Características das Crianças}

No que diz respeito à idade das crianças hospitalizadas, a média de idades é de 3,13 anos $(D P=1,895)$, variando entre 1 mês de vida e os 14 anos.
Relativamente ao número de irmãos, este varia entre nenhum e três, verificando-se que 20 das crianças são filhos únicos $(42,6 \%)$, número que se repete para as crianças que tem pelo menos um irmão. Neste estudo é pouco representativo o número mais elevado de irmãos.

\section{Características da Hospitalização}

Passando agora aos dados específicos da hospitalização, verifica-se que a maioria das crianças não tinha tido hospitalizações anteriores (85,1\%) o que está associado ao tipo de patologia e início da doença, sendo de aparecimento súbito em $83,0 \%$ dos casos.

\section{Escalas de Ansiedade, Depressão e Stress (EADS)}

Analisando o quadro $\mathrm{n}^{\circ} 1$, que reporta para a análise descritiva da nossa amostra, verificamos estar perante uma distribuição normal, uma vez que os valores de Skewness (assimetria) e Kurtosis (achatamento) encontram-se dentro do intervalo ] -1,1 [. Consequentemente, foram utilizados testes paramétricos para efectuar as análises estatísticas consideradas como pertinentes para a compreensão da problemática em causa.

A resposta mais representativa das mães face à hospitalização das crianças, foi o stresse $(M=10,30$; $D P=$ $4,288)$, seguindo-se a ansiedade $(M=8,38$; $D P=5,156)$ e por último a depressão $(\mathrm{M}=8,11 ; \mathrm{DP}=5,503)$.

Quadro $n^{\circ} 1$ - Análise das variáveis EADS-21

\begin{tabular}{ccccc}
\hline Variável & $M$ & $D P$ & Skewness & Kurtosis \\
Ansiedade & 8,38 & 5,156 &, 362 &,- 950 \\
Depressão & 8,11 & 5,503 &, 473 &,- 873 \\
Stress & 10,30 & 4,288 &, 202 &,- 192 \\
\hline
\end{tabular}

\section{Relação da Idade das Mães com as Variáveis Dependentes}

Categorizando a variável independente idade da mãe aplicou-se o One Way Anova para comparação de médias nas variáveis dependentes: ansiedade, depressão e stress. As mães com idades compreendidas entre os 26 e 36 anos, obtém médias mais altas para o stresse $(M=10,97$, com $D P=$ $3,980)$ e ansiedade $(M=9,17 ; \mathrm{DP}=5,176)$, com excepção para os sintomas de depressão, que apresenta uma média mais elevada na faixa etária superior, dos 36 aos 46 anos (M $=9,88$, com $\mathrm{DP}=5,793$ ). (quadro 2 ).

Quadro ${ }^{\circ} 2$ - Análise comparativa das variáveis por grupos de idade das mães

\begin{tabular}{lccccc}
\hline & $16-26$ anos & $26-36$ anos & $36-46$ anos & $>46$ anos & $p$ \\
& $M \pm D P$ & $M \pm D P$ & $M \pm D P$ & $M \pm D P$ &, 149 \\
Ansiedade & $4,80 \pm 2,280$ & $9,17 \pm 5,176$ & $8,71 \pm 5,499$ & $1,00 \pm-$ &, 261 \\
Depressão & $3,60 \pm 2,881$ & $8,81 \pm 5,414$ & $9,88 \pm 5,793$ & $1,00 \pm-$ &, 898 \\
Stresse & $7,83 \pm 3,545$ & $10,97 \pm 3,980$ & $10,63 \pm 4,689$ & $1,00 \pm-$ & \\
\hline
\end{tabular}




\section{Relação da Escolaridade das Mães com as Variáveis Dependentes}

Correlacionando a variável da escolaridade da mãe, com as nossas variáveis dependentes (ansiedade, depressão e stresse), verificou-se, em todas elas, uma média mais elevada nas mães que concluíram o $3^{\circ}$ ciclo do ensino básico (stresse $M=11,91$; ansiedade $M=9,70$; depressão $M=9,45$, quadro 3).

Estes resultados foram obtidos a partir do teste One Way Anova, não se encontrando diferenças estatisticamente significativas.

Quadro $n^{\circ} 3$ - Análise comparativa das variáveis pelas habilitações literárias das mães

\begin{tabular}{lcccccc}
\hline & $1^{\circ}$ ciclo & $2^{\circ}$ ciclo & $3^{\circ}$ ciclo & Secundário & Superior & $p$ \\
& $M \pm D P$ & $M \pm D P$ & $M \pm D P$ & $M \pm D P$ & $M \pm D P$ &, 621 \\
Ansiedade & $9,50 \pm 2,726$ & $6,63 \pm 5,181$ & $9,70 \pm 5,397$ & $6,71 \pm 5,499$ & $8,78 \pm 6,457$ &, 841 \\
Depressão & $8,00 \pm 4,528$ & $6,44 \pm 5,151$ & $9,45 \pm 6,121$ & $8,33 \pm 4,457$ & $8,10 \pm 6,967$ &, 611 \\
Stresse & $10,56 \pm 3,358$ & $9,00 \pm 3,428$ & $11,91 \pm 4,949$ & $9,38 \pm 4,749$ & $10,20 \pm 4,803$ &, 612 \\
\hline
\end{tabular}

\section{Relação do Estado Civil das Mães com as Variáveis Dependentes}

Da análise do quadro $n^{\circ} 4$, observa-se que o stresse continua a ser a dimensão percepcionada como sendo a mais afectada, com uma média mais elevada para o estado civil de viuvez $(\mathrm{M}=12,00)$. As mães casadas são as que apresentam valores mais elevados de ansiedade $(M=8,67$, com $D P=5,259)$. Isto poderá indicar que os níveis de comprometimento, responsabilidade, aliados à pressão social, podem influenciar positivamente a ansiedade das mães. Por sua vez, o estado civil, divorciada tem uma correlação mais elevada na depressão $(M=10,00$, com $D P=5,431)$. Estes resultados foram obtidos a partir do teste One Way Anova, não se encontrando diferenças estatisticamente significativas.

Quadro $n^{\circ} 4$ - Análise comparativa das variáveis pelo estado civil das mães

\begin{tabular}{ccccc}
\hline & Casada & Divorciada & Viuva & $p$ \\
& $M \pm D P$ & $M \pm D P$ & $M \pm D P$ &, 716 \\
Ansiedade & $8,67 \pm 5,259$ & $7,20 \pm 4,817$ & $4,00 \pm-$ &, 450 \\
Depressão & $7,85 \pm 5,603$ & $10,00 \pm 5,431$ & $9,00 \pm-$ &, 389 \\
Stresse & $10,40 \pm 4,488$ & $9,33 \pm 3,204$ & $12,00 \pm-$ & \\
\hline
\end{tabular}

\section{Relação da Residência das Mães com as Variáveis Dependentes}

Para análise dos resultados relativos às variáveis ansiedade, depressão e stresse com a residência das mães, realizou-se o T-test de Student, onde mais uma vez não existe diferenças estatisticamente significativas entre as variáveis dependentese a residência das mães. Verificou-se uma média de stresse mais elevado nas mães que vivem em meio urbano (Média=11,05, com $\mathrm{DP}=4,488)$ comparativamente às que vivem em meio rural (Média $=9,74$, com $\mathrm{DP}=4,166$ ), ao contrário da ansiedade (Média=8,67, com DP=5,088) e depressão (Média=8,19, com $\mathrm{DP}=6,020)$ com médias superiores para as mães que vivem em meio rural (quadro 5).
Quadro $n^{\circ} 5$ - Análise comparativa das variáveis pela residência das mães

\begin{tabular}{lccc}
\hline & Urbano & Rural & $p$ \\
& $M \pm D P$ & $M \pm D P$ & $p$ \\
Ansiedade & $8,00 \pm 5,369$ & $8,67 \pm 5,088$ &, 831 \\
Depressão & $8,00 \pm 4,865$ & $8,19 \pm 6,020$ &, 055 \\
Stresse & $11,05 \pm 4,442$ & $9,74 \pm 4,166$ &, 900 \\
\hline
\end{tabular}

\section{Relação da Situação Profissional das Mães com as} Variáveis Dependentes

Correlacionando as variáveis dependentes com a situação profissional da mãe, constata-se pela análise do quadro 6 , que as mães desempregadas, relativamente às mães empregadas são as que apresentam pontuações mais satisfatórias nas três dimensões da EADS-21 (apesar das diferenças não serem estatisticamente significativas, com a aplicação do T-test). O stresse foi o sintoma mais representativo, com uma média de 10,50, a ansiedade 8,89 e a depressão 8,85 .

Quadro $n^{\circ} 6$ - Análise comparativa das variáveis pela situação profissional das mães

\begin{tabular}{cccc}
\hline & Empregada & Desempregada & $p$ \\
Ansiedade & $M \pm D P$ & $M \pm D P$ & \\
Depressão & $8,00 \pm 4,881$ & $8,89 \pm 5,603$ &, 523 \\
Stresse & $7,52 \pm 5,189$ & $8,85 \pm 5,923$ &, 531 \\
\hline
\end{tabular}

\section{Relação da Idade das Crianças com as Variáveis Dependentes}

Um outro dado interessante refere-se à idade das crianças hospitalizadas. Nesta variável, o grupo de crianças com mais de 12 anos (apesar de as diferenças não serem significativas com realização teste One Way Anova), é aquele que apresenta pontuações mais satisfatórias nas dimensões ansiedade $(M=10,29 ; D P=5,908)$ e depressão $(M=10,38$; $\mathrm{DP}=6,423)$ da EADS-21. A dimensão stresse tem uma média mais elevada de 11,43 para as mães de crianças na faixa etária dos 9-12 anos (quadro 7).

Quadro $n^{\circ} 7$ - Análise comparativa das variáveis por grupos de idade das crianças

\begin{tabular}{lccccccc}
\hline & $<1$ ano & $1-3$ anos & $3-6$ anos & $6-9$ anos & $9-12$ anos & $>12$ anos & $p$ \\
& $M \pm D P$ & $M \pm D P$ & $M \pm D P$ & $M \pm D P$ & $M \pm D P$ & $M \pm D P$ & $p$ \\
Ansiedade & $8,36 \pm 5,887$ & $8,67 \pm 3,775$ & $6,75 \pm 7,544$ & $5,75 \pm 2,630$ & $8,57 \pm 5,224$ & $10,29 \pm 5,908$ &, 259 \\
Depressão & $6,38 \pm 4,700$ & $8,10 \pm 5,801$ & $8,00 \pm 6,928$ & $6,33 \pm 4,041$ & $9,57 \pm 5,711$ & $10,38 \pm 6,413$ &, 772 \\
Stresse & $10,62 \pm 4,646$ & $10,10 \pm 3,291$ & $9,80 \pm 3,633$ & $8,25 \pm 2,630$ & $11,43 \pm 5,381$ & $10,38 \pm 5,528$ &, 530 \\
\hline
\end{tabular}

\section{Relação do Número de Irmãos da Criança com as Variáveis Dependentes}

Compararam-se os valores de depressão, ansiedade e stresse com o número de irmãos das crianças hospitalizadas, pela análise do quadro 8 verificou-se que a dimensão stresse tem uma média mais elevada, de 12,50 nas mães em que as crianças têm mais 2 irmãos. A dimensão depressão apresenta uma média de 8,37 nas mães em que as crianças têm apenas 1 irmão. 
$\mathrm{Na}$ ansiedade, ao contrário das outras dimensões, temos uma média mais elevada $(M=9,44 ; D P=4,938)$ nas mães de filho único, ou seja o filho que está hospitalizado.

Quadro n 8 - Análise comparativa das variáveis por número de irmãos das crianças

\begin{tabular}{cccccc}
\hline & nenhum & 1 & 2 & 3 & $p$ \\
& $M \pm D P$ & $M \pm D P$ & $M \pm D P$ & $M \pm D P$ & \\
Ansiedade & $9,44 \pm 4,938$ & $8,12 \pm 5,7970$ & $6,50 \pm 4,509$ & $6,00 \pm 3,464$ &, 592 \\
Depressão & $7,89 \pm 5,675$ & $8,37 \pm 5,927$ & $8,00 \pm 2,160$ & $8,00 \pm 7,211$ &, 995 \\
Stresse & $10,80 \pm 4,086$ & $9,60 \pm 4,806$ & $12,50 \pm 3,109$ & $8,67 \pm 3,055$ &, 527 \\
\hline
\end{tabular}

\section{Relação do Inicio da Doença com as Variáveis Dependentes}

O quadro 9 representa a comparação entre as médias das variáveis dependentes com a forma como a doença se manifestou nas crianças, aplicou-se o T-test de Student onde mais uma vez não se verificou diferenças estatisticamente significativas. Constatou-se que a dimensão stresse e a depressão têm uma média mais elevada nas manifestações de doença de início insidioso (stresse $M=12,50 ; D P=5,767$ e depressão $M=9,29$; $D P=7,017$ ) ao passo que a ansiedade tem valores mais elevados na doença de início súbito $(M=$ $8,41 ; \mathrm{DP}=4,924)$.

Quadro $n^{\circ} 9$ - Análise comparativa das variáveis por início da doença da criança

\begin{tabular}{cccc}
\hline & Súbito & Insidioso & $p$ \\
& $M \pm D P$ & $M \pm D P$ & $p$ \\
Ansiedade & $8,41 \pm 4,924$ & $8,25 \pm 6,431$ &, 379 \\
Depressão & $7,89 \pm 5,265$ & $9,29 \pm 7,017$ &, 253 \\
Stresse & $9,85 \pm 3,877$ & $12,50 \pm 5,767$ &, 071 \\
\hline
\end{tabular}

Relação de Hospitalizações Anteriores da Criança com as Variáveis Dependentes

Um outro dado analisado refere-se à experiência das mães face a hospitalizações anteriores do filho doente. Nesta variável, o grupo que referiu ter tido experiencias com hospitalizações anteriores do filho doente (apesar de as diferenças não serem significativas com realização T-test de Student), é aquele que apresenta pontuações mais satisfatórias nas dimensões stresse e depressão da EADS-21 (stresse $M=$ $10,71$ e $\mathrm{DP}=4,889$ e depressão $\mathrm{M}=9,29$ e $\mathrm{DP}=6,525)$. $\mathrm{Na}$ ansiedade o mesmo não sucedeu, as mães sem experiência de hospitalizações do filho apresentaram pontuações mais elevadas, com uma média de 8,66 (quadro 10).

Quadro $n^{\circ} 10$ - Análise comparativa das variáveis por hospitalizações anteriores da criança

\begin{tabular}{|c|c|c|c|}
\hline & $\begin{array}{c}\operatorname{sim} \\
M \pm D P\end{array}$ & $\begin{array}{c}N a ̃ o \\
M \pm D P\end{array}$ & $p$ \\
\hline Ansiedade & $7,00 \pm 5,802$ & $8,66 \pm 5,064$ & 967 \\
\hline Depressão & $9,29 \pm 6,525$ & $7,89 \pm 5,367$ &, 559 \\
\hline Stresse & $10,71 \pm 4,889$ & $10,23 \pm 4,240$ & ,646 \\
\hline
\end{tabular}

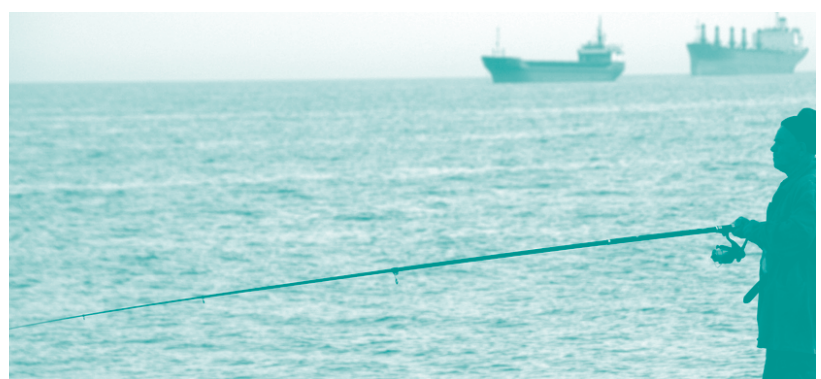

\section{DISCUSSÃO DOS RESULTADOS}

Os dados obtidos indicam que, tal como a literatura sugere, a hospitalização da criança desencadeia sentimentos de insegurança, medo, depressão, stresse e ansiedade, provocando uma crise emocional na família, sendo consideradas reacções normais e adaptativas (Ferreira, 2010; Jorge, 2004; Pedro, 2009).

As mulheres casadas apresentam uma maior incidência de doenças mentais (ansiedade, depressão e stresse), do que as solteiras, exceptuando-se as mulheres separadas ou divorciadas (Silva,1999). Estes dados estão de acordo com o estudo, as mães casadas foram as que apresentaram uma média mais elevada de ansiedade, as mães divorciadas apresentaram uma média maior na depressão e o stresse esteve mais presente nas mães viúvas.

De acordo com os resultados verificou-se que ser mãe mais velha está associado ao facto de apresentar mais sintomas depressivos (Baião, 2009).

As mães com menos escolaridade apresentam ainda, índices de ansiedade, depressão e stresse. No entanto estes dados vem confirmar os resultados de outros estudos, onde relacionam as habilitações literárias e respostas humanas (ansiedade, stresse e depressão), concluindo-se que as últimas assumem-se de forma mais expressiva em elementos da amostra que possuem habilitações literárias iguais ou inferiores ao 9o ano de escolaridade (Silva, 1999). Numa tentativa de explicação deste facto, Vaz Serra (2002) refere que o grau de instrução determina comummente a forma como a pessoa se descreve. Se o grau de educação é elevado as queixas tendem a ser «intelectualizadas», enquanto os grupos iletrados incidem a sua atenção nas perturbações físicas.

Viver em meio rural, fora da abrangência do hospital provoca nas mães índices de ansiedade e de depressão mais elevados. $O$ facto de as mães não residirem na zona de abrangência do hospital influencia positivamente o seu estado emocional (Jorge, 2004).

A profissão parece ser protectora para as manifestações psíquicas, estatutos sócioeconómicos mais desfavorecidos resultam em níveis mais elevados de stresse, ansiedade e depressão (Baião, 2009). Estes dados reforçam os resultados do estudo, em que as mães desempregadas, com estatutos socioeconómicos mais desfavorecidos, foram as que apresentaram maiores níveis de stresse, ansiedade e depressão.

Verificou-se, contrariamente ao esperado, que as mães de crianças mais velhas são as que apresentam maiores índices de ansiedade, depressão e stresse. O mesmo sucedeu num estudo realizado por Baião, 2009, em que as mães de crianças mais velhas revelaram, níveis de stresse mais elevados em comparação com as mães de bebés. Tendo 
em conta a literatura, os resultados obtidos poderão estar relacionados com o estado clínico favorável dos bebés-alvo.

O facto de ter mais filhos associa-se a um maior número de acontecimentos stressantes, os resultados estão de acordo com os estudos (Baião, 2009; Jorge, 2004), em que ter mais filhos associa-se a um maior número de acontecimentos stressantes. A hospitalização da criança traz dificuldades essencialmente a nível familiar e, mais relacionadas com o filho doente e os outros filhos, imaginando como suporte a família nuclear e de origem (Jorge, 2004). Foi possível verificar neste estudo que as grandes fontes de stresse na família foram os filhos doentes, o hospitalizado e os outros.

As crianças hospitalizadas por doença de início súbito provocaram nas mães níveis mais elevados de ansiedade, contrariamente à depressão e stresse que se relacionam com doenças de início insidioso. Nas doenças de início súbito, as mudanças comportamentais e afectivas requerem dos pais e família uma rápida mobilização das suas competências para lidar com a situação de crise, enquanto nas doenças de aparecimento prolongado existe um período de maior adaptação (Jorge, 2004). Estes dados vêm confirmar os resultados de um estudo realizado por Pereira e Lopes (2005), em que os autores referem que após o primeiro impacto do diagnóstico da doença, os pais passam por uma fase tipicamente caracterizada por um período de ansiedade, raiva, protesto, associado com sentimentos de culpabilização.

A fase de início insidioso, corresponde neste estudo às doenças crónicas, nesta fase ocorre o reconhecimento de que a condição de doença crónica existe. Nesta fase podem surgir sentimentos de depressão, desespero e isolamento, no qual prevalece e encontra-se exacerbado um sentimento de vulnerabilidade e solidão associado a um sentido marcado de perda (Pereira \& Lopes, 2005). Nesta fase de doença foram manifestados sentimentos de stresse por parte das mães, pressupõe-se que esteja relacionado com o facto de uma nova confrontação com o processo de hospitalização. A experiência da família de doença e/ou hospitalização, pode conduzir a situação de crise ou numa situação de crescimento ou maturação da família (Pedro, 2009).

\section{CONCLUSÕES}

A hospitalização da criança é um importante aconteci-mmento na vida familiar podendo apresentar-se como uma emergência, atendendo a que a família necessita de ajuda externa para se proteger de uma mudança não desejada. Pode ser o ponto de partida para a crise, quando o auxíllio externo não é suficiente para evitar a necessidade de uma mudança qualitativa.

O modo como a família se adapta à situação de ter uma criança doente e hospitalizada, depende dos seus próprios recursos intrínsecos, bem como de suportes familiares e sociais disponíveis. A atenção passa por conhecer os seus medos, dificuldades, sentimentos e necessidades, respeitando o conhecimento parental da criança e o seu direito a participar na tomada de decisões e no processo de prestação de cuidados.

As respostas dos pais à hospitalização dependem da gravidade e eminência de ameaça ao filho doente, capaci-ndade de mobilização dos recursos familiares, experiên $\neg$ cias anteriores, crenças e valores e outros. A culpa é uma resposta quase universal para os pais (Jorge, 2004).

Os cuidadas de enfermagem devem dar resposta as necessidades cognitivas, emocionais, comportamentais dos pais. Para estes, é importante: ver que os filhos estão a receber os cuidados físicos competentes, compreender a situação clínica, sentir que são importantes para os seus filhos e capazes e que tenham oportunidade de discutir os sentimentos sobre a hospitalização.

O papel do enfermeiro na ajuda aos pais passa pelo reconhecimento e a descoberta dos seus potenciais recursos, de acordo com a sua própria personalidade, para a resolução dos seus problemas. A ajuda só será eficaz quando o enfermeiᄀro interiorizar que só o ajudado possui os recursos base para resolver o seu problema; este, apenas deve orientar e assistir e nunca decidir ou substituir no processo de resolução de problemas. Assim, a ajuda faz com que haja no outro crescimenᄀto, desenvolvimento, maturidade, um melhor funcionamento e uma maior capacidade para enfrentar a vida (Chalifour, 1993; Lazure, 1994). Com a noção de relação subentende-se a presen $\neg$ ça de elos de contactos, de uma forma de coexistência.

\section{REFERÊNCIAS BIBLIOGRÁFICAS}

Baião, Rute (2009). Stress Parental e Prematuridade [Texto policopiado]. Lisboa: Universidade de Lisboa: Faculdade de Psicologia e Ciências de Educação. Dissertação de Mestrado em Psicologia.

Bicho, D; Pires, A. (2002) Comportamento de mães de crianças hospitalizadas devido a queimaduras. Análise Psicológica, 1 (XX): 115-129.

Chalifour, J. (1993). La relation d'aide eu soins infirmiers: une perspective holistique-humaniste. Montreal: Gâetan Morin Editeur Itée.

Ferreira, P. [et al]. (2010). Validação e análise da precisão da versão em português do Needs of Parents Questionnaire. Jornal de Pediatria. Vol. 86, № 3, p. 221-227.

Gomes, P. (1999). A criança e a Nova Pediatria. Lisboa: Fundação Calouste Gulbenkian.

Jorge, A. (2004). Família e Hospitalização da Criança - (Re) Pensar o Cuidado em Enfermagem. Loures: Lusociência.

Lazure, H. (1994). Viver a relação de ajuda: abordagem teórica 
e prática de um critério de competência de enfermagem. Lisboa: Lusodidacta.

Marinheiro, P. (2002). Enfermagem de Ligação. Coimbra: Quarteto.

Moleiro, A.; et al (1991). Humanizar o atendimento à criança. Lisboa. Secção de Pediatria Social da Sociedade Portuguesa de Pediatria.

Mota, A. (2000). Psiquiatria de Ligação. Medicina Interna Vol. 7, N. 4.

Pedro, J. (2009). Parceiros no Cuidar: A perspectiva do Enfermeiro no cuidar com a família, a criança com doença crónica [texto policopiado]. Porto: Instituto de Ciências Biomédicas de Abel Salazar. Dissertação de Mestrado em Ciências de Enfermagem.

Pereira, M. \& Lopes, C. (2005). O doente oncológico e a sua família: Manuais Universitários. $2^{a}$ Edição. Lisboa: Climepsi Editores.

Reis, G. \& Santos, M. (1996). A importância da presença dos pais na unidade de cuidados intensivos do hospital pediátrico de Coimbra. Sinais vitais, 8: 25-28.

Ribeiro, J; Honrado, A. \& Leal, I. (2004). Contribuição para o estudo da adaptação Portuguesa das escalas de Ansiedade, Depressão e Stress (EADS) de 21 itens de Lovibond e Lovibond. Psicologia, Saúde \& Doenças, Ano/vol. V, número 002. Sociedade Portuguesa de Psicologia da Saúde. Lisboa, Portugal, 229-239.

Silva, M. F.(1999). Diferenças de género na depressão e desempenho profissional [texto policopiado]. Porto: Faculdade de Medicina da Universidade do Porto. Dissertação apresentada com vista à obtenção do Grau de Mestre, no âmbito do V Curso de Mestrado em Psiquiatria e Saúde Mental.

Vaz Serra, A. (2002). O stress na vida de todos os dias. Coimbra: Gráfica de Coimbra.

Viana, V; et al (2005), Apoio às mães em crise num Serviço de Neonatologia. Psicologia, Saúde \& Doenças, Ano/vol. VI, número 002. Sociedade Portuguesa de Psicologia da Saúde. Lisboa, Portugal, 119-130.

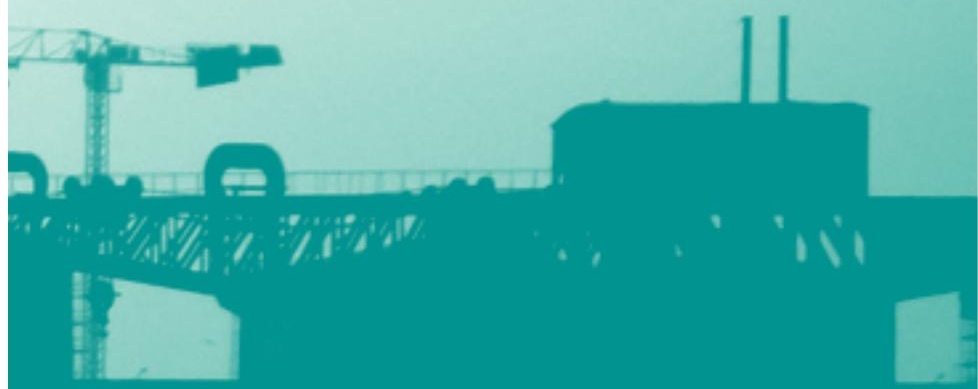

